\title{
Triple combination therapy with telmisartan, amlodipine, and hydrochlorothiazide ameliorates albuminuria in a normotensive rat remnant kidney model
}

Sakuya Ito ${ }^{1}$, Yosuke Nakayama ${ }^{1}$, Goh Kodama ${ }^{1}$, Kanji Ota ${ }^{1}$, Kensei Taguchi ${ }^{1}$, Yusuke Kaida', Sho-ichi Yamagishi ${ }^{2}$ and Kei Fukami ${ }^{1 *}$ (D)

\begin{abstract}
Background: Some types of antihypertensive drugs may have pleiotropic effects in patients with chronic kidney disease (CKD). However, whether triple combination therapy with angiotensin II receptor blockers (ARBs), calcium channel blockers (CCBs), and thiazide diuretics (TZD) confer renoprotective effects in normotensive CKD remains unknown. Thus, we explored this issue using a normotensive rat remnant kidney model.
\end{abstract}

Methods: Sprague-Dawley rats were randomly allocated into four groups: sham $(n=10), 5 / 6$ nephrectomy (NTx) ( $n$ $=9$ ), NTx treated with telmisartan and amlodipine (dual) $(n=8)$, and NTx treated with telmisartan, amlodipine, and hydrochlorothiazide (triple) $(n=7)$, and followed for 4 weeks. Blood pressure (BP), blood chemistry including renal function, urinary albumin excretion (UAE), and renal pathology were evaluated in all groups.

Results: There was no significant change in systolic BP among the four groups during the study period. Serum blood urea nitrogen (BUN) was significantly higher, and 24-h creatinine clearance (Ccr) was lower in all NTx groups $(p<0.001)$. Dual therapy further increased the glomerular diameter in NTx rats $(p<0.001)$, which was significantly ameliorated by triple therapy $(p<0.001)$. Triple therapy, but not dual therapy, significantly reduced NTx-induced UAE levels $(p<0.05)$, whereas BUN, 24-h Ccr, and tubulointerstitial injury scores were comparable among all the NTx groups.

Conclusions: Our results suggest that triple combination therapy with telmisartan, amlodipine, and hydrochlorothiazide could ameliorate glomerular hypertrophy and albuminuria in normotensive CKD rats in a BPlowering independent manner.

Keywords: Thiazide diuretics, RAS inhibitor, Ca channel blocker, Renal injury, CKD

\footnotetext{
* Correspondence: fukami@med.kurume-u.ac.jp

'Division of Nephrology, Department of Medicine, Kurume University School of Medicine, 67 Asahi-machi, 6, Fukuoka 830-0011, Japan

Full list of author information is available at the end of the article
}

(C) The Author(s). 2021 Open Access This article is licensed under a Creative Commons Attribution 4.0 International License, which permits use, sharing, adaptation, distribution and reproduction in any medium or format, as long as you give appropriate credit to the original author(s) and the source, provide a link to the Creative Commons licence, and indicate if changes were made. The images or other third party material in this article are included in the article's Creative Commons. licence, unless indicated otherwise in a credit line to the material. If material is not included in the article's Creative Commons licence and your intended use is not permitted by statutory regulation or exceeds the permitted use, you will need to obtain permission directly from the copyright holder. To view a copy of this licence, visit http://creativecommons.org/licenses/by/4.0/. The Creative Commons Public Domain Dedication waiver (http://creativecommons.org/publicdomain/zero/1.0/) applies to the data made available in this article, unless otherwise stated in a credit line to the data. 


\section{Introduction}

Chronic kidney disease (CKD) is a major contributor to the increased risk of morbidity and mortality worldwide [1]. The Kidney Disease Improving Global Outcome Clinical Practice Guideline for the Management of Blood Pressure in CKD has announced that renin-angiotensin system inhibitors (RAS-i) are the first-line therapy for not only decreasing blood pressure (BP) but also preventing the progression of renal dysfunction in CKD patients with albuminuria and diabetes $[2,3]$. There are several combination therapies for treating patients with hypertension. However, compared with others, a combination of angiotensin II type-1 receptor blockers (ARBs) and calcium channel blockers (CCBs) has more renoprotective effects in CKD patients. Indeed, ARB reduces urinary protein excretion and has better renoprotective effects in normotensive patients with CKD without decreasing both diastolic and systolic BP [4]. Furthermore, combination therapy with RAS-i and CCB has been proven to decrease all-cause and cardiovascular mortality as well as major cardiovascular events, especially in high-risk patients such as CKD patients [5, 6]. However, whether these combinations could contribute to the amelioration of renal injury independent of BP is unknown.

The effects of thiazide diuretics (TZDs) on renal injury in patients with CKD are controversial. Chlorthalidone, one of the TZDs, significantly decreased the change in glomerular filtration rate (GFR) compared with other agents such as angiotensin-converting enzyme (ACE) inhibitors and $\mathrm{CCB}$ for 4 years [7]. Furthermore, combining $\mathrm{ACE}$ inhibitor with $\mathrm{CCB}$ was superior to combining the same ACE inhibitor with hydrochlorothiazide (HCTZ) in slowing the progression of renal dysfunction in patients at high risk for cardiovascular events [8]. However, these studies did not focus on the pleiotropic effects of combination therapy with TZD on renal function. In contrast, in an in vivo study, HCTZ was reported to reduce BP, renal vascular resistance, and glomerular capillary pressure, and the combination of RAS-i with HCTZ further decreased the $\mathrm{BP}$ and glomerular pressure in $N^{\omega}$-nitro-L-arginine methyl ester-treated spontaneously hypertensive rats (LNAME/SHR), an animal model of CKD. These findings led us to investigate whether triple combination therapy with RAS-i, CCB, and/or HCTZ could directly contribute to renal protection in a CKD model independent of BP.

To clarify this issue, we examined the effects of combination therapy involving $\mathrm{ARB}$ and $\mathrm{CCB}$ with or without HCTZ on renal involvement in a normotensive subtotal nephrectomy (NTx) rat model.

\section{Materials and methods}

\section{Experimental design}

Seven-week-old male Sprague-Dawley (SD) rats (200$250 \mathrm{~g})$ were purchased from Charles River Laboratory,
Japan, Inc. The rats were randomly divided into four groups: the sham $(n=10), \mathrm{NTx}(n=9), \mathrm{NTx}$ treated with telmisartan and amlodipine (dual) $(n=8)$, and NTx treated with telmisartan, amlodipine, and hydrochlorothiazide (triple) $(n=7)$ groups. When the rats were 8 weeks old, NTx was performed as described previously [9]. In brief, the right kidney was removed, and 1 week later, the lower and upper thirds of the left kidney were resected. In this study, CKD was conducted by resecting the kidney $5 \mathrm{~mm}$ bilaterally from the center of the kidney, as shown in Fig. 1.

Subsequently, we investigated the efficacies of dual therapy with telmisartan $(3.2 \mathrm{mg} / \mathrm{kg} /$ day $)$ and amlodipine $(0.2 \mathrm{mg} / \mathrm{kg} /$ day $)$ and triple therapy with telmisartan, amlodipine, and HCTZ $(0.5 \mathrm{mg} / \mathrm{kg} /$ day $)$ on BP and renal derangements in NTx rats for 4 weeks (Fig. 1). To avoid drug-induced hypotension and renal injury, the doses of drugs were set to twice the maximum dose for humans. Carboxymethylcellulose (CMC) was used as a vehicle for these drugs. These antihypertensive drugs were administered by gavage once daily.

All rats were placed in metabolic cages for $24 \mathrm{~h}$ for urinalysis during overnight fasting. BP was measured with a tail-cuff sphygmomanometer using an automated system with a photoelectric sensor (BP-98A; Softron, Tokyo, Japan) before surgery and 2 and 4 weeks after treatment. Finally, the rats were anesthetized with 99\% diethyl ether (Nacalai Tesque, Kyoto, Japan) and sacrificed with a decapitator after overnight fasting. Serum samples were obtained, and the residual kidney was removed. The kidney was weighted and fixed with $10 \%$ buffered formalin for histological analysis. All experimental procedures were conducted in accordance with the National Institutes of Health Guide for the Care and Use of Laboratory Animals and were approved by the ethical committee of Kurume University School of Medicine.

\section{Biochemical analysis}

Serum levels of creatinine $(\mathrm{Cr})$, blood urea nitrogen (BUN), serum sodium, potassium, calcium, and phosphate were measured using an auto-analyzer (Nihondenshi Co., Tokyo, Japan). Urinary albumin excretion (UAE) was evaluated using a commercially available enzyme-linked immunosorbent assay kit (Exocell, Philadelphia, USA). Creatinine clearance (Ccr) was calculated using the following formula: 24-h Ccr (mL/ $\mathrm{min} / 100 \mathrm{~g} \mathrm{BW})=($ urinary creatinine $\times$ urinary volume $(\mathrm{mL} /$ day $) /($ serum $\mathrm{Cr} \times 24 \times 60)$ /body weight [10]

\section{Renal histological analysis}

The kidney was obtained from each rat, cut transversally, fixed in Bouin's solution followed by $10 \%$ buffered formalin, and embedded in paraffin. Four-micrometer 
(The removal portion of the left kidney)
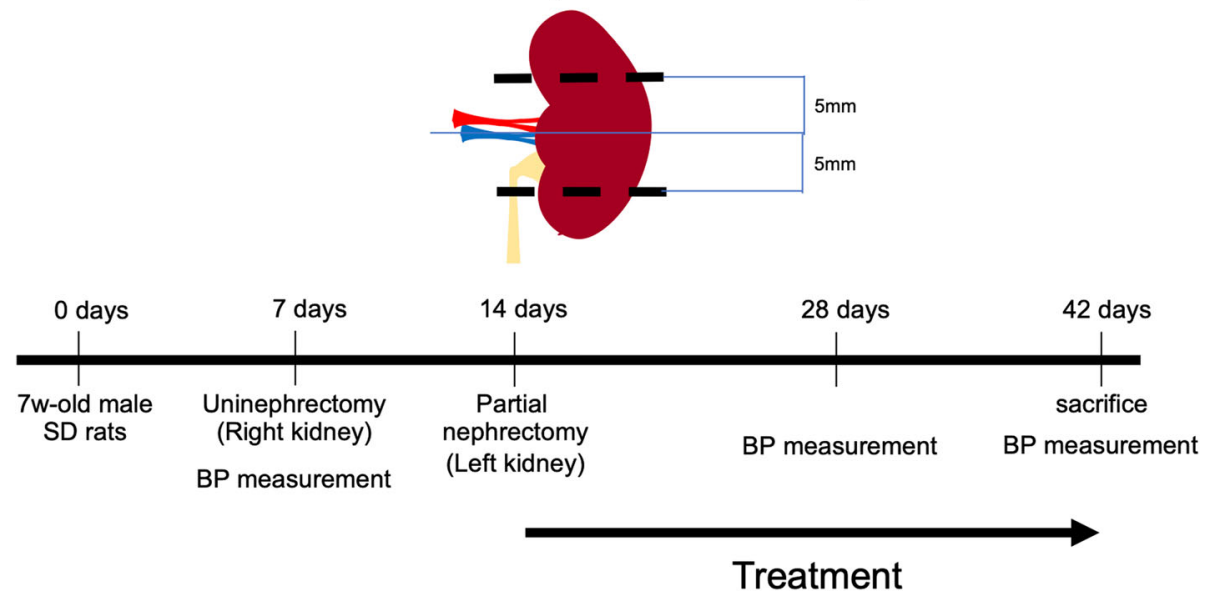

Fig. 1 Protocol for the removal of the left kidney in CKD rats. Abbreviations: CKD, chronic kidney disease; SD, Sprague-Dawley; BP, blood pressure

paraffin sections were stained with periodic acid-Schiff and Masson's trichrome to examine the diameter of glomeruli and tubulointerstitial injury (TI) score. Fifty glomeruli per rat residual kidney were evaluated to determine the diameter of the glomeruli. The TI score was evaluated using the intensity of the blue color in the cortex after staining with Masson's trichrome without glomeruli and medulla and divided by the area of the renal specimen using the BZ-X800 Analyzer software (Keyence, Osaka, Japan).

\section{Statistical analysis}

All data are presented as mean \pm standard error of the mean (SEM). One-way analysis of variance followed by post hoc multiple comparison analysis (Tukey-Kramer test) was used to assess the differences among the groups if the variances were equal. Non-parametric analysis (Steal-Dwass test) was used if the data did not show equal variances. The variance was analyzed using the Leven and Bartlett tests. The unpaired Student's $t$ test was used to compare the BP between the two groups. All statistical analyses were performed using the JMP Pro software ver. 14 (SAS Institute Inc., NC, USA). Statistical significance was set at $p<0.05$.

\section{Results}

Effects of combination therapy on clinical and blood chemistry in rats with subtotal nephrectomy

Heart rate and body weight were not significantly different between the groups (Table 1). Systolic BP levels were not changed in the NTx groups as well as in NTx rats that underwent dual and triple therapy after 0,2 , and 4 weeks (Fig. 2a). Renal function evaluated by 24-h Ccr and BUN significantly worsened in all the NTx rats

Table 1 Characteristics of all groups 4 weeks after treatment

\begin{tabular}{lllll}
\hline & Sham & NTx + CMC & NTx + T + A & NTx + T +A + H \\
\hline Number & 10 & 9 & 8 & 7 \\
HR (/min) & $381 \pm 16$ & $385 \pm 17$ & $399 \pm 18$ & $383 \pm 19$ \\
Systolic BP (mmHg) & $117 \pm 4$ & $115 \pm 4$ & $106 \pm 5$ & $108 \pm 5$ \\
Body weight (g) & $460 \pm 11$ & $430 \pm 12$ & $486 \pm 13^{\#}$ & $442 \pm 13$ \\
BUN (mg/dL) & $16.2 \pm 1.5$ & $29.4 \pm 1.6^{* * *}$ & $27.8 \pm 0.7^{* * *}$ & $27.0 \pm 1.8^{* * *}$ \\
Na (mmol/L) & $138 \pm 2$ & $142 \pm 1$ & $137 \pm 2$ & $134 \pm 2^{\#}$ \\
K (mmol/L) & $5.84 \pm 0.16$ & $5.56 \pm 0.17$ & $5.05 \pm 0.18^{* * . \#}$ & $5.64 \pm 0.19$ \\
Calcium (mg/dL) & $9.88 \pm 0.15$ & $9.92 \pm 0.16$ & $9.82 \pm 0.17$ & $9.43 \pm 0.18$ \\
Phosphate (mg/dL) & $6.84 \pm 0.24$ & $7.16 \pm 0.25$ & $6.60 \pm 0.27$ & $6.97 \pm 0.29$ \\
Urinary Na (nmol/day) & $754 \pm 57$ & $680 \pm 60$ & $427 \pm 64^{* *}$ & $681 \pm 68$ \\
\hline
\end{tabular}

Data are shown as mean \pm SEM

Abbreviations: NTX nephrectomized, $H R$ heart rate, CMC carboxymethylcellulose, $T$ telmisartan, $A$ amlodipine, $H$ hydrochlorothiazide, $B U N$ blood urea nitrogen, Na sodium, $K$ potassium

${ }^{* *} p<0.01,{ }^{* * *} p<0.001$ vs sham, ${ }^{*} p<0.05$ vs NTx + CMC 

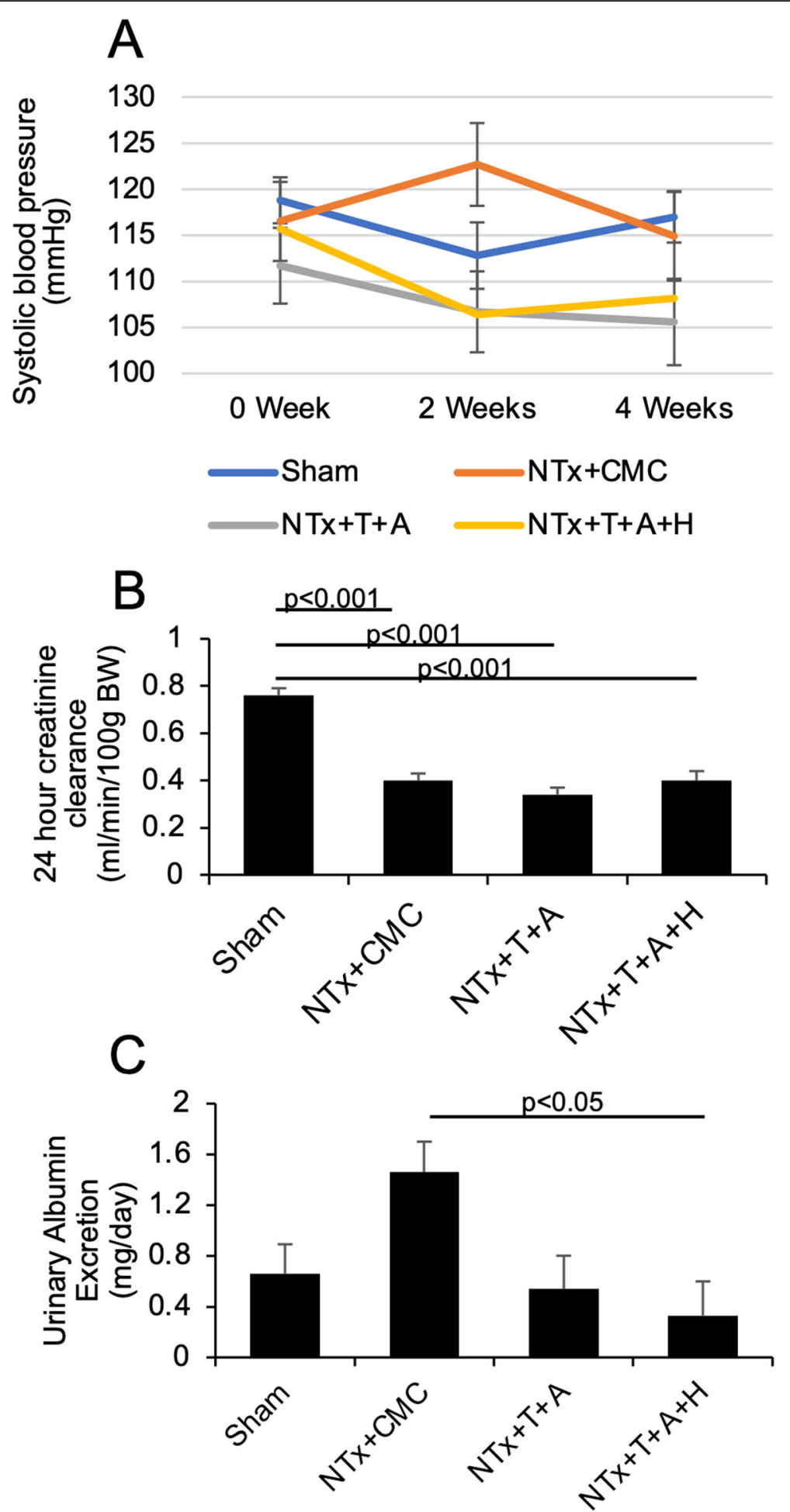

Fig. 2 Effects of dual and triple therapies on blood pressure, 24-h creatinine clearance, and albuminuria in CKD rats. a Systolic blood pressure before and after the treatments. b, c Twenty-four-hour Ccr levels. d, e Urinary albumin excretion levels. Abbreviations: CKD, chronic kidney disease; NTx, nephrectomy; Ccr, creatinine clearance; CMC, carboxymethylcellulose; BW, body weight; T, telmisartan; A, amlodipine; $H$, hydrochlorothiazide 
compared with sham-operated rats and were not improved by the dual or triple combination therapy (Fig. $2 \mathrm{~b}$ and Table 1). Non-treated NTx rats showed no changes in the serum or urinary electrolytes. However, dual therapy decreased serum potassium and urinary sodium excretion levels, which were returned by the triple combination therapy (Table 1).

\section{Effects of dual and triple combination therapies on albuminuria and renal pathology in rats with nephrectomy}

UAE levels tended to increase in non-treated NTx rats. The increase was significantly attenuated by the triple therapy (sham $0.66 \pm 0.23$, NTx + CMC $1.46 \pm 0.24$, dual therapy $0.54 \pm 0.26$, triple therapy $0.33 \pm 0.27 \mathrm{mg} /$ day, NTx + CMC vs triple therapy $p<0.05$ ) (Fig. 2c). The diameter of glomeruli was increased in NTx + CMC rats compared with sham-operated rats, which was further deteriorated by dual therapy, but not by triple therapy in NTx rats (sham $123.5 \pm 2.7$, NTx +
CMC $150.3 \pm 2.6$, dual therapy $173.2 \pm 2.6$, triple therapy $154.4 \pm 2.6 \mu \mathrm{m}, p<0.001$, respectively) (Fig. 3a, b). TI score was higher in NTx + CMC rats compared with sham-operated rats. The score did not improve in rats following dual and triple combination therapies (sham $1.21 \pm 0.56$, NTx + CMC $3.80 \pm 0.56$, dual therapy $4.23 \pm 0.59$, triple therapy $3.69 \pm 0.63 \%$, sham vs NTx $p<0.01$ ) (Fig. 3c, d).

\section{Discussion}

RAS-i is a first-line antihypertensive therapy for CKD patients with proteinuria and is used to prevent the progression of renal dysfunction $[11,12]$. Combinations of antihypertensive drugs are necessary to achieve optimal $\mathrm{BP}$ control and ameliorate the progression of renal dysfunction in patients with CKD [13]. The most common combination of antihypertensive agents in patients with CKD is the combination of RAS-i and CCB. RAS-i ameliorated glomerular hyperfiltration and albuminuria in hypertensive CKD patients, and L-type CCB, including
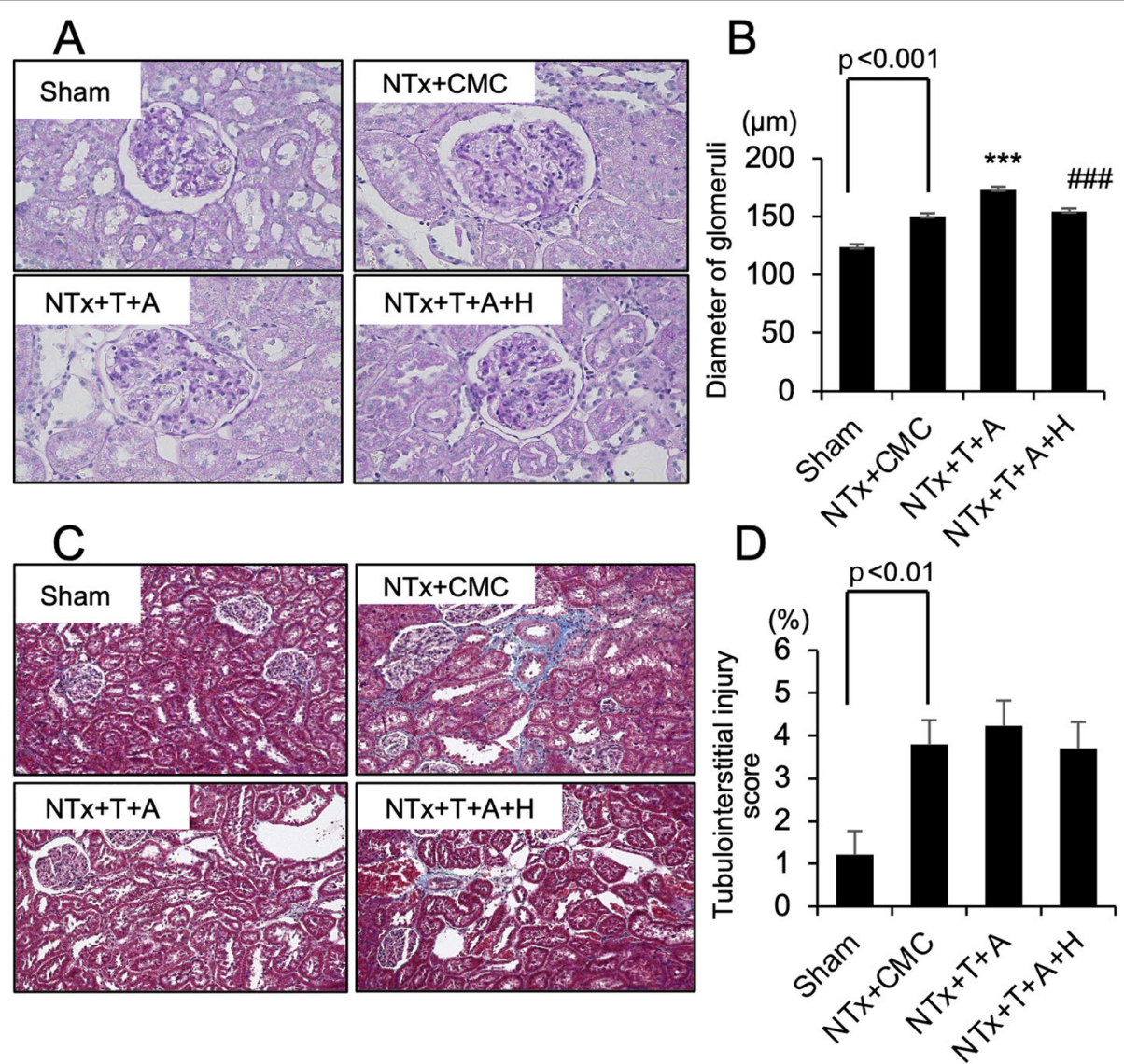

Fig. 3 Effects of dual and triple therapies on glomerular diameter and tubulointerstitial injury in CKD rats. a Representative images of the glomeruli following periodic acid-Schiff staining $(\times 400)$. b Quantitative graph of the diameter of the glomeruli. c Representative images of the tubulointerstitium following Masson's trichrome staining $(\times 200)$. d Quantitative graph of the interstitial injury score. ${ }^{* * *} p<0.001$ vs sham, ${ }^{\# \# \# ~} p$ < 0.001 vs NTx+T+A. Abbreviations: CKD, chronic kidney disease; NTx, nephrectomy; CMC, carboxymethylcellulose; T, telmisartan; A, amlodipine; $H$, hydrochlorothiazide 
amlodipine, demonstrated a protective role through the vasodilation of glomerular arterioles in 5/6 NTx spontaneously hypertensive rats fed a high-salt diet [14]. Glomerular diameter has been reported to increase in some pathological conditions such as obesity-related renal disease, focal segmental glomerulosclerosis, diabetic nephropathy, and cyanotic kidney disease. However, the protective effects of combination therapies against normotensive CKD remain unclear. Therefore, we examined the effects of combination therapy on glomerular hypertrophy in the NTx model, which mimics normotensive and non-metabolic CKD patients with hyperfiltration. Dual therapy tended to reduce albuminuria but significantly increased glomerular diameter in normotensive NTx rats. This discrepancy might be due to the changes in glomerular hemodynamics and antioxidant effects of $\mathrm{CCB}$ and $\mathrm{ARB}$ in normotensive conditions. RAS-i reduces glomerular hyperfiltration through the inhibition of angiotensin II-induced vasoconstriction in glomerular efferent arterioles, whereas CCB dilates glomerular arterioles, leading to neutral glomerular pressure in hypertensive patients. In this study, however, the effects of CCB on the increase in glomerular filtration might be greater than the effects of $A R B$, thereby increasing renal plasma flow without glomerular hypertension under normotensive conditions. The decrease in albuminuria might be due to the RAS-iassociated antioxidant effects. Further experiments involving the direct measurement of glomerular pressure and oxidative stress measurement in vivo are necessary to clarify this mechanism.

Most patients recruited to investigate the effects of antihypertensive drugs on cardiovascular events and renal involvement in large clinical trials had already been prescribed with RAS-i and TZD $[12,15]$. However, the effects of combination therapy with TZD on renal function in patients with CKD remain controversial. In this study, triple, but not dual therapy, significantly reduced UAE in normotensive NTx rats without any changes in $\mathrm{BP}$, electrolytes, or renal function. It has been reported that TZD combined with losartan attenuates glomerular overload and albuminuria, indicating that this dual therapy may provide glomerular protection in patients with elevated glomerular pressure without causing prolonged damage to the kidney [16]. Moreover, dual concomitant use of telmisartan and TZD resulted in a significantly greater decrease in albuminuria, which is independent of BP, compared with telmisartan alone [17]. Furthermore, it has been reported that HCTZ has direct vascular relaxant effects through modulation of the opening of $\mathrm{Ca}$ activated potassium channels [18]. HCTZ combined with RAS-i reduces the mean arterial pressure, total peripheral resistance, glomerular hydrostatic pressure, and afferent and efferent glomerular arteriolar resistances in
L-NAME/SHR [19]. Accordingly, the direct vasodilating effects of HCTZ combined with telmisartan and amlodipine might reduce the intraglomerular pressure, thereby preventing glomerular diameter increase and albuminuria in NTx CKD rats. In this study, triple therapy tended to decrease body weight and increase urinary sodium excretion compared with dual therapy in CKD rats. Although the main mechanism of action of TZD during organ protection is related to the reduction of $\mathrm{BP}$ via the elimination of sodium and extra fluid, natriuresis-induced glomerular hemodynamics by HCTZ, independent of BP, might also be involved in the prevention of kidney damage.

In conclusion, triple combination therapy with telmisartan, amlodipine, and HCTZ improved NTx-induced enlargement of glomerular diameter and albuminuria compared to dual combination therapy with telmisartan and amlodipine in normotensive CKD rats. Further clinical and experimental studies are required to clarify the mechanism underlying the beneficial effects of triple combination therapy in normotensive CKD patients.

\section{Abbreviations \\ ACE: Angiotensin-converting enzyme; ARB: Angiotensin II type-1 receptor blocker; BP: Blood pressure; BUN: Blood urea nitrogen; CCB: Calcium channel blocker; Ccr: Creatinine clearance; CKD: Chronic kidney disease; \\ CMC: Carboxymethylcellulose; Cr: Creatinine; GFR: Glomerular filtration rate; HCTZ: Hydrochlorothiazide;" L-NAME/SHR: $N^{\omega}$-nitro-L-arginine methyl ester- treated spontaneously hypertensive rats; NTx: Subtotal nephrectomy; RAS- i: Renin-angiotensin system inhibitors; SD: Sprague-Dawley; \\ TI: Tubulointerstitial injury; TZD: Thiazide diuretics; UAE: Urinary albumin excretion}

\section{Acknowledgements}

This work was supported, in part, by Grants-in-Aid for Welfare and Scientific Research (C) (no. 19k08693) (K.F) and from the Ministry of Education, Culture, Sports, Science and Technology of Japan.

\section{Authors' contributions}

$\mathrm{SI}, \mathrm{YN}, \mathrm{GK}, \mathrm{KO}$, and KT managed the animals, collected the samples, and performed the histological studies. YK and KF organized the study protocol. SY and KF wrote the manuscript. The authors read and approved the final manuscript.

\section{Availability of data and materials}

The datasets used and/or analyzed during the current study are available from the corresponding author upon reasonable request.

\section{Declarations}

Ethics approval and consent to participate

This article does not contain any studies involving human participants.

\section{Competing interests}

Telmisartan, amlodipine, and hydrochlorothiazide were supplied by Boehringer Ingelheim. K.F. and Y.S. received honoraria, such as lecture fees and research funding from Boehringer Ingelheim Co., Ltd.

\section{Author details}

'Division of Nephrology, Department of Medicine, Kurume University School of Medicine, 67 Asahi-machi, 6, Fukuoka 830-0011, Japan. ${ }^{2}$ Division of Diabetes, Metabolism, and Endocrinology, Department of Medicine, Showa University School of Medicine, Tokyo, Japan. 
Received: 9 September 2020 Accepted: 29 March 2021

Published online: 09 April 2021

\section{References}

1. Carney EF. The impact of chronic kidney disease on global health. Nat Rev Nephrol. 2020;16(5):251. https://doi.org/10.1038/s41581-020-0268-7.

2. KDIGO Clinical Practice Guideline for the Management of Blood Pressure in Chronic Kidney Disease. Chapter 3: Blood pressure management in CKD ND patients without diabetes mellitus. Kidney Int Suppl (2011). 2012;2(5):357-62. https://doi.org/10.1038/kisup.2012.53.

3. KDIGO Clinical Practice Guideline for the Management of Blood Pressure in Chronic Kidney Disease. Chapter 4: Blood pressure management in CKD ND patients with diabetes mellitus. Kidney Int Suppl (2011). 2012;2(5):363-9. https://doi.org/10.1038/kisup.2012.54.

4. Geng DF, Sun WF, Yang L, En G, Wang JF. Antiproteinuric effect of angiotensin receptor blockers in normotensive patients with proteinuria: a meta-analysis of randomized controlled trials. J Renin Angiotensin Aldosterone Syst. 2014;15(1):44-51. https://doi.org/10.11 77/1470320312474054.

5. Mourad JJ, Le Jeune S, Pirollo A, Mourad C, Gaudouen Y, Lopez-Sublet M. Combinations of inhibitors of the renin-angiotensin system with calcium channel blockers for the treatment of hypertension: focus on perindopril/ amlodipine. Curr Med Res Opin. 2010;26(9):2263-76. https://doi.org/10.1185/ 03007995.2010.510925.

6. Locatelli F, Del Vecchio L, Andrulli S, Colzani S. Role of combination therapy with ACE inhibitors and calcium channel blockers in renal protection. Kidney Int Suppl. 2002;82:S53-60. https://doi.org/10.1046/j.1523-1755.62. s82.11.x

7. Trial AOaCftACRGTAaL-LTtPHA. Major outcomes in high-risk hypertensive patients randomized to angiotensin-converting enzyme inhibitor or calcium channel blocker vs diuretic: the Antihypertensive and Lipid-Lowering Treatment to Prevent Heart Attack Trial (ALLHAT). JAMA. 2002;288(23):298197. https://doi.org/10.1001/jama.288.23.2981.

8. Bakris GL, Sarafidis PA, Weir MR, Dahlöf B, Pitt B, Jamerson K, et al. Renal outcomes with different fixed-dose combination therapies in patients with hypertension at high risk for cardiovascular events (ACCOMPLISH): a prespecified secondary analysis of a randomised controlled trial. Lancet. 2010;375(9721):1173-81. https://doi.org/10.1016/ S0140-6736(09)62100-0.

9. Matsumoto Y, Ueda S, Yamagishi S, Matsuguma K, Shibata R, Fukami K, et al. Dimethylarginine dimethylaminohydrolase prevents progression of renal dysfunction by inhibiting loss of peritubular capillaries and tubulointerstitial fibrosis in a rat model of chronic kidney disease. J Am Soc Nephrol. 2007; 18(5):1525-33. https://doi.org/10.1681/ASN.2006070696.

10. Takamiya Y, Fukami K, Yamagishi SI, Kaida Y, Nakayama Y, Obara N, et al. Experimental diabetic nephropathy is accelerated in matrix metalloproteinase-2 knockout mice. Nephrol Dial Transplant. 2013;28(1):5562. https://doi.org/10.1093/ndt/gfs387.

11. Lewis EJ, Hunsicker LG, Clarke WR, Berl T, Pohl MA, Lewis JB, et al. Renoprotective effect of the angiotensin-receptor antagonist irbesartan in patients with nephropathy due to type 2 diabetes. N Engl J Med. 2001; 345(12):851-60. https://doi.org/10.1056/NEJMoa011303.

12. Brenner BM, Cooper ME, de Zeeuw D, Keane WF, Mitch WE, Parving HH, et al. Effects of losartan on renal and cardiovascular outcomes in patients with type 2 diabetes and nephropathy. N Engl J Med. 2001;345(12):861-9. https://doi.org/10.1056/NEJMoa011161.

13. Weir MR. The role of combination antihypertensive therapy in the prevention and treatment of chronic kidney disease. Am J Hypertens. 2005; 18(4 Pt 2):100S-5S. https://doi.org/10.1016/j.amjhyper.2004.11.017.

14. Saruta T, Kanno Y, Hayashi K, Konishi K. Antihypertensive agents and renal protection: calcium channel blockers. Kidney Int Suppl. 1996;55: S52-6.

15. Wright JT, Williamson JD, Whelton PK, Snyder JK, Sink KM, Rocco MV, et al. A randomized trial of intensive versus standard blood-pressure control. N Engl J Med. 2015;373(22):2103-16. https://doi.org/10.1056/ NEJMoa1511939.

16. Hasegawa H, Tayama Y, Takayanagi K, Asakura J, Nakamura T, Kawashima K, et al. Release from glomerular overload by the addition of low-dose thiazide in patients with angiotensin receptor blocker-resistant hypertension. Kidney Blood Press Res. 2013;37(6):521-30. https:/doi.org/1 $0.1159 / 000355732$
17. Ekinci El, Thomas G, Thomas D, Johnson C, Macisaac RJ, Houlihan CA, et al. Effects of salt supplementation on the albuminuric response to telmisartan with or without hydrochlorothiazide therapy in hypertensive patients with type 2 diabetes are modulated by habitual dietary salt intake. Diabetes Care 2009;32(8):1398-403. https://doi.org/10.2337/dc08-2297.

18. Calder JA, Schachter M, Sever PS. Direct vascular actions of hydrochlorothiazide and indapamide in isolated small vessels. Eur J Pharmacol. 1992;220(1):19-26. https://doi.org/10.1016/0014-2999(92)90006-P.

19. Zhou X, Matavelli LC, Ono H, Frohlich ED. Superiority of combination of thiazide with angiotensin-converting enzyme inhibitor or AT1-receptor blocker over thiazide alone on renoprotection in L-NAME/SHR. Am J Physiol Renal Physiol. 2005;289(4):F871-9. https://doi.org/10.1152/ajprena I.00129.2005.

\section{Publisher's Note}

Springer Nature remains neutral with regard to jurisdictional claims in published maps and institutional affiliations.
Ready to submit your research? Choose BMC and benefit from:

- fast, convenient online submission

- thorough peer review by experienced researchers in your field

- rapid publication on acceptance

- support for research data, including large and complex data types

- gold Open Access which fosters wider collaboration and increased citations

- maximum visibility for your research: over $100 \mathrm{M}$ website views per year

At BMC, research is always in progress.

Learn more biomedcentral.com/submissions 